\title{
Krieg und Zwangsarbeit im pharaonischen Ägypten
}

\author{
Von \\ RiCHARD BUSSMANN
}

Das pharaonische Ägypten entstand im ausgehenden Neolithikum am Ende des 4. Jahrtausends v. Chr. und blickte auf eine dreitausendjährige Geschichte zurück, als es 30 v. Chr. in das römische Reich eingegliedert wurde. Schon Herodot, der das Land im 5. Jahrhundert v. Chr. bereist hat, beschrieb die ägyptische Kultur als sehr alt und exotisch. Die Fremdartigkeit des alten Ägypten übt auch heute noch eine ungebrochene Faszination auf den Betrachter aus, fordert aber zur Umsicht in der historischen Analyse von Phänomenen wie Krieg und Zwangsarbeit auf. Angesichts von Tempeln und Pyramiden liegt es nahe, vom pharaonischen Ägypten das Bild einer Gesellschaft zu zeichnen, deren Herrschaftselite die eigene Bevölkerung und Kriegsgefangene zu Repräsentationszwecken ausbeutete und zur Zwangsarbeit verpflichtete. Die deutlichsten Worte dafür fand der Sinologe und Soziologe Karl Wittfogel. In seiner kulturvergleichenden Studie Oriental Despotism, die 1957 erstmals veröffentlicht wurde, beschrieb er Krieg und Zwangsarbeit in der pharaonischen Gesellschaft vor dem Hintergrund der Gewalterfahrungen des Zweiten Weltkrieges und des Aufstiegs von Faschismus und Kommunismus. ${ }^{1}$ Wittfogels Werk wurde in der Ägyptologie jedoch nur schwach rezipiert. Für Befremden sorgten offensichtliche empirische Mängel und die unkritische Projektion zeitgenössischer Erfahrungen in den archäologischen Befund einer vergangenen Hochkultur.

Gleichwohl wirft eine epochenübergreifende und transglobale Perspektive die Frage auf, wie weit sich der Zusammenhang von bewaffneten Konflikten und Zwangsarbeit historisch zurückverfolgen lässt. Das pharaonische Ägypten nimmt hierfür eine Schlüsselposition ein. Sozialevolutionistische Theorien zählen es zu den frühen komplexen Gesellschaften der Weltgeschichte, die sich durch neue Formen der Arbeitsteilung und eine neue Rationalität von Herrschaft auszeichnen. ${ }^{2}$ Im westlichen Verständnis markiert der Beginn der pha-

Karl August Wittfogel, Oriental despotism. A comparative study of total power, New Haven/ London 1957. Danach sei Ägypten der frühe Vertreter einer hydraulischen Gesellschaft, in der sich Herrschaft auf die Kontrolle der Wasserwirtschaft stütze und auf die ausbeuterische »asiatische Produktionsweise « gründe. Zu Wittfogels Biographie und seiner durch zeitgenössische Erfahrungen geprägte Sichtweise vgl. Joachim Radkau, Der Emigrant als Warner und Renegat: Karl August Wittfogels Dämonisierung der »asiatischen Produktionsweise«, in: Exilforschung. Ein internationales Jahrbuch 1 (1983), S. 73-94.

2 Jospeh A. Tainter, The nature of complex societies, in: Joseph A. Tainter (Hrsg.), The collapse of complex societies, Cambridge 1988, S. 22-31; Bruce G. Trigger, Understanding early civilizations. A comparative study, Cambridge 2003, S. 40-52; Norman Yoffee, Myths of the Archaic State. Evolution of the Earliest Cities, States, and Civilizations, Cambridge 2005, S. 4-21. 
raonischen Geschichte die Schwelle von der Vor- und Frühgeschichte zu unserer »historischen « Zeit und gilt als eine Phase der frühen Staatenbildung. ${ }^{3}$ In der Ägyptologie besteht im Wesentlichen Konsens darüber, dass der pharaonische Staat aus kriegerischen Auseinandersetzungen hervorgegangen ist und für den Bau von Pyramiden und Tempeln auf eine Form der »Zwangsarbeit« (corvée) zurückgriff. Danach würden Staat, Krieg und Zwangsarbeit bis an den Ursprung der »historischen « Zeit zurückreichen und wären das Ergebnis wachsender sozialer Komplexität.

Zweifellos steht auch hier der moderne westliche Staat Pate für die Modellbildung und leitet den Blick auf die pharaonische Gesellschaft. Angesichts der brüchigen und in mehrfacher Hinsicht verzerrten Quellenlage ist es methodisch ausgesprochen schwierig, nicht der Gefahr zu erliegen, zeitgenössische Begriffe, Sachverhalte und Deutungsmuster in die Analyse eingehen zu lassen. Ziel des vorliegenden Beitrags ist es, Krieg und Zwangsarbeit als Triebkräfte sozialen Wandels darzustellen und die relevanten Befunde quellenkritisch im gesellschaftlichen Kontext ihrer Zeit zu verorten.

Der wohl bekannteste Hinweis auf die Existenz von Zwangsarbeit im pharaonischen Ägypten ist der alttestamentarische Bericht in den ersten Kapiteln des Buches Exodus (1,11-14; 5,5-19). Danach wurden die Israeliten mit Gewalt zur Feldarbeit und zum Heranschaffen von Tonziegeln für den Bau der Stadt »Ramses« gezwungen. Archäologisch und ikonographisch lassen sich dafür stimmige Korrelate im ägyptischen Material anführen (Abb. 1). ${ }^{4}$ Spätestens seit dem dritten Jahrtausend gab es an der Nordostgrenze Ägyptens militärische Auseinandersetzungen mit nicht-ägyptischen Bevölkerungsgruppen. ${ }^{5}$ Der ägyptischen Quellenlage nach könnte es sich bei den Israeliten daher um Kriegsgefangene des Neuen Reichs (1550-1070 v. Chr.) handeln. ${ }^{6}$ In einer Inschrift Amenhoteps III. (1388-1350 v. Chr.) heißt es: »[...] Die Bauhütte des Tempels ist angefüllt mit Sklaven und Sklavinnen (äg. hem) und mit Kindern der Anführer aller Fremdländer der Eroberung Seiner Majestät. [...] Der Tempel ist umgeben von Syrer-Siedlungen, die von den Kindern der Großen ge-

Zusammenfassend E. Christiana Köhler, Theories of State Formation, in: Willemina Wendrich (Hrsg.), Egyptian Archaeology, Oxford/Malden/Chichster 2010, S. 36-54.

4 Mafred Bietak/Irene Forstner-Müller, The Topography of New Kingdom Avaris and Per-Ramesses, in: Mark Collier/Steven Snape (Hgg.), Ramesside Studies in Honour of K.A. Kitchen, Bolton 2011, S. 23-50.

5 Donald B. Redford, Egypt, Canaan, and Israel in Ancient Times, Princeton 1992. Mit neuem archäologischen Material: James K. Hoffmeier, The »Walls of the Ruler«. Egyptian Literature and the Archaeological Record: Investigating Egypt's Eastern Frontier in the Bronze Age, in: Bulletin of the American Schools of Oriental Research 343 (2006), S. 1-20; Ann E. Killebrew, Biblical peoples and ethnicity. An archaeological study of Egyptians, Canaanites, Philistines, and early Israel, 1300-1100 B.C.E., Altanta 2005.

6 Bernadette Menu, Captifs de guerre et dépendance rurale dans l'Égypte du Nouvel Empire, in: Bernadette Menu (Hrsg.), La dépendance rurale dans l' Antiquité égyptienne et proche-orient, Kairo 2001, S. 187-205. 
gründet worden sind. « Eine der historischen Sequenzen des Großen Papyrus Harris (col. 77,4-6) von Ramses III. (1182-1151 v. Chr.) lautet: »[...] Ich habe [die besiegten Feinde nach Ägypten] gebracht [...] zusammen mit unzähligen Kriegsgefangenen. [...] Ihre Frauen und ihre Kinder waren Zehntausende, ihr Kleinvieh aller Art waren Hunderttausende. Ich habe ihre besten Soldaten in Festungen mit meinem Namen stationiert. Ich habe ihnen zahlreiche Bogentruppenaufseher gegeben, [...] nachdem sie gestempelt, zu Sklaven (äg. hem) gemacht und mit meinem Namen gekennzeichnet worden sind. Ihre Frauen und Kinder wurden genauso behandelt. ${ }^{8}$

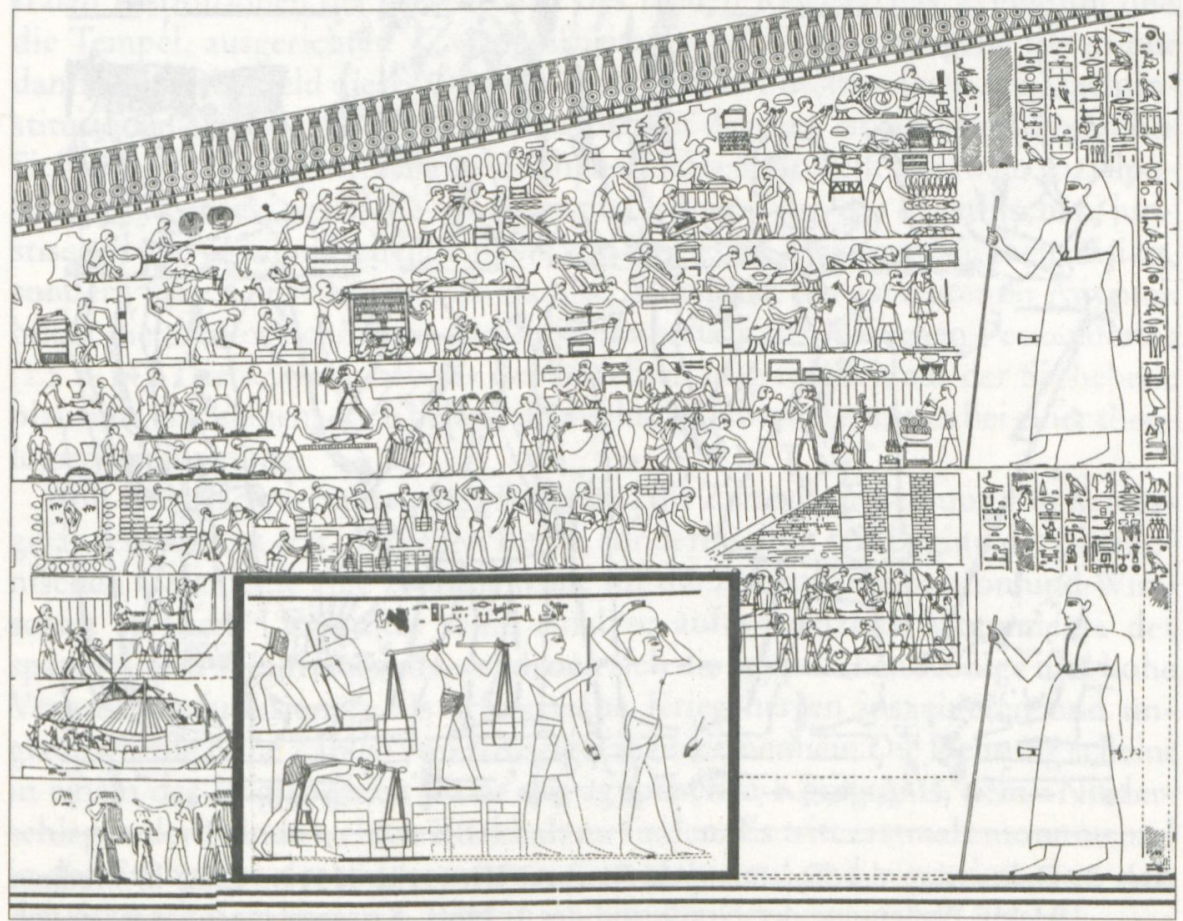

Abb. 1: Grabdekoration des Wesirs Rechmire, Theban Tomb 100, ca. 1400 v. Chr. Rechmire beaufsichtigt verschiedene Arbeiten, darunter die Lehmziegelherstellung, 4. Register von unten. In dem vergrößerten Abschnitt des Registers ist ein Aufseher, der einen Arbeiter mit einem Stock antreibt, zu sehen. Die Arbeiter werden dort als »Kriegsgefangene, die Seine Majestät für die Arbeiten des Amuntempels hergebracht hat « bezeichnet. Nach Norman de Garris Davies, Paintings from the tomb of Rekh-mi-Rec, New York 1935, pl. 23 und ders., The tomb of Rekh-mi-Rec at Thebes, New York 1943, pl. 59.

Wilhelm Spiegelberg, Die Bauinschrift Amenophis III. auf der Flinders Petrie-Stele, in: Receuil de Travaux relatifs à Philologie et à Archéologie Égyptiennes et Assyriennes 20 (1890), S. 37-54.

8 Pierre Grandet, Papyrus Harris I (BM 9999), Kairo 1994, Band I, S. 337, Band II, S. 252-254. 
Wie die Israeliten setzen sich die in diesen beiden Texten beschriebenen Gruppen von Kriegsgefangenen aus Männern, Frauen und Kindern zusammen, die vermutlich als Familien in eigenen, vielleicht separierten Siedlungen wohnten. Andere Kriegsgefangene wurden als Soldaten für das ägyptische Heer rekrutiert. Die Beschriftung, vielleicht Brandmarkung, fremder Soldaten durch ägyptische Militärbeamte ist auch auf einem Wandrelief des Totentempels Ramses III. dargestellt (Abb. 2). Die Tempel waren in dieser Epoche offenbar die zentrale Institution, die den Einsatz der Kriegsgefangen koordinierte und regelte.

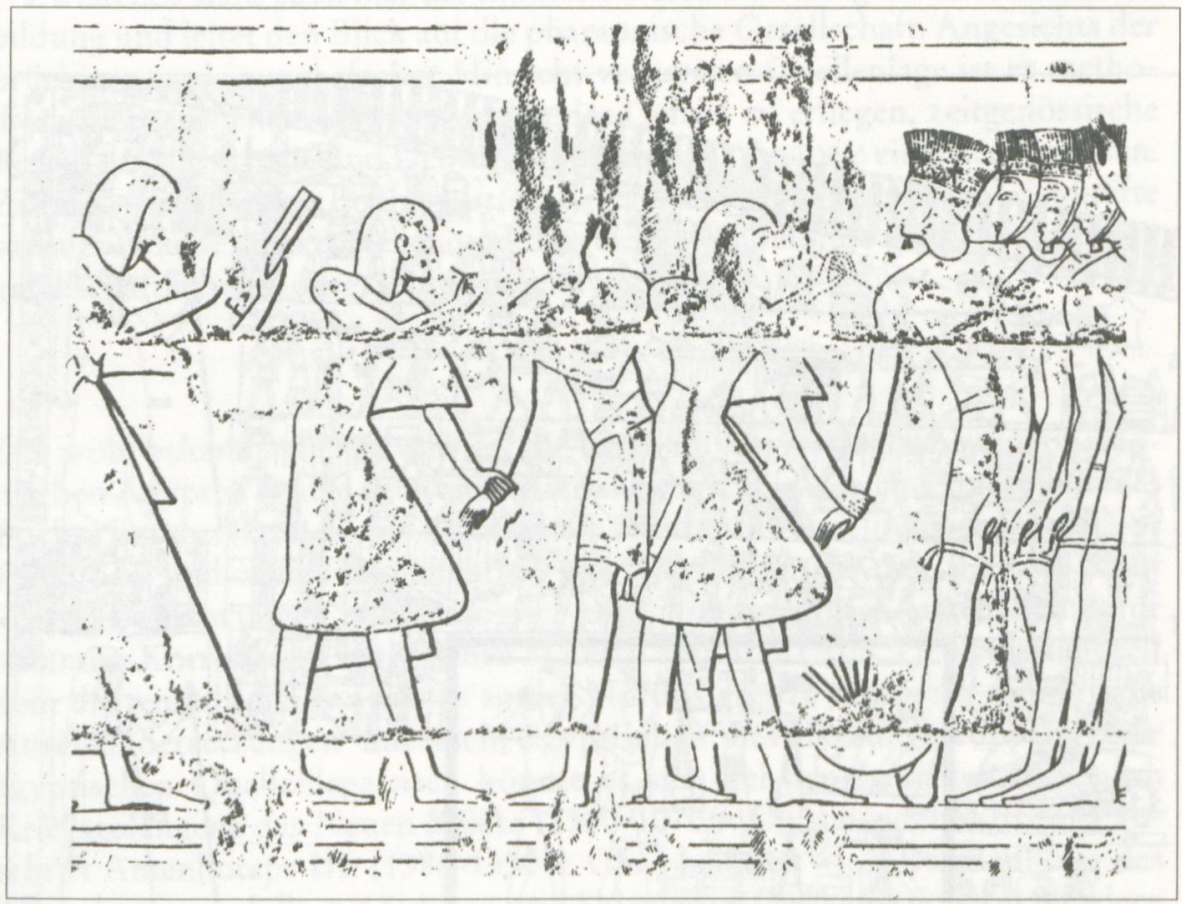

Abb. 2: Dekoration des Totentempels Ramses' III. (1182-1151 v. Chr.) in Medinet Habu, Außenseite der Nordwand des 1. Hofs. Kriegsgefangene werden von ägyptischen Militärbeamten beschriftet oder gebrandmarkt. Nach Bernadette Menu, Captifs de guerre et dépendance rurale dans l'Égypte du Nouvel Empire, in: Bernadette Menu (Hrsg.), La dépendance rurale dans l'Antiquité égyptienne et proche-orient, Kairo 2001, fig. 6.

Das Alte Testament ist jedoch eine umstrittene Geschichtsquelle, da die biblischen Texte über Jahrhunderte fortgeschrieben wurden. ${ }^{9}$ Auch im philologischen Detail offenbart sich die Notwendigkeit zur Quellenkritik. ${ }^{10}$ Die Luther-

Werner H. Schmidt, Exodus 1 - 6, Neukirchen-Vluyn 1988, S. 1-48; William H. C. Propp, Exodus 1-18. A New Translation with Introduction and Commentary, New York, London, Toronto, Sydney, Auckland 1999, S. 125-127.

10 W. Dietrich, Sklaverei I. Altes Testament, in: Gerhard Müller (Hrsg.), Theologische Realenzyklopädie, Berlin, New York 2000, S. 367-373. 
Übersetzung von 1984 gibt das hebräische סלות (Ex 1,11), etymologisch "Tragearbeit«, mit "Zwangsarbeit« wieder, die deutsche Einheitsübersetzung van 1980 spricht von »schwerer Arbeit «. Das drei Verse später verwendete עבדה (Ex 1,14) erscheint in der Luther-Übersetzung als »Frondienst «, in der Einheitsübersetzung als »Sklavenarbeit«. Das Beispiel zeigt, dass zeitgenössische Erfahrungen und Semantiken in die Lesart des Textes eingegangen sind und dessen Verständnis manipulieren. ${ }^{11}$

Schwerwiegender als die Übersetzungsproblematik ist, wessen Perspektive die unterschiedlichen Quellen vermitteln. Die oben zitierten ägyptischen Schrift- und Bildquellen dienen der Elitenrepräsentation und sind auf die zentralen Institutionen der Gesellschaft des Neuen Reiches, das Königtum und die Tempel, ausgerichtet. "Zwangsarbeiter « und Kriegsgefangene treten nur dann in das Blickfeld dieser Quellen, wenn sie das Funktionieren der Machtinstitutionen garantieren. Der biblische Bericht dagegen ist aus ägyptologischer Sicht ein seltenes Schriftzeugnis der Beherrschten. Er stellt aber kein sozialpolitisches Manifest gegen die herrschende Ordnung dar, das die ethischen, juristischen und ökonomischen Aspekte von Krieg und Zwangsarbeit thematisiert, sondern folgt seiner eigenen Logik: der Aufenthalt der Israeliten in Ägypten bildet die theologische Folie für die Verankerung des jüdischen Pessachfestes (Ex 8-14). Die »Zwangsarbeit« der Israeliten mag folglich auf der Sachebene plausibel erscheinen, doch belässt die biblische Überlieferung es bei einer theologischen Deutung.

Rolf Gundlach vermutet, dass Krieg und die Zwangsumsiedlung von Kriegsgefangenen nicht erst im Neuen Reich, sondern schon seit Beginn der pharaonischen Geschichte eine zentrale Rolle für die Arbeitsorganisation und Wirtschaft spielten. ${ }^{12}$ Er stützt seine Analyse auf vereinzelte Monumente des späten 4. und 3. Jahrtausends, auf denen sich die ägyptischen Könige und hohe Verwaltungsfunktionäre als erfolgreiche Kriegsherren inszenieren und unglaubwürdig hohe Zahlen von Kriegsgefangenen nennen. Die Deutung scheint in einem der langlebigsten Ikone des ägyptischen Königtums, dem »Niederschlagen der Feinde«, einen Rückhalt zu finden. Es tritt erstmals monumental in der Frühphase der pharaonischen Geschichte auf und suggeriert, dass sich Ägypten seit dem späten 4. Jahrtausend im Dauerkrieg befand (Abb. 3).

11 Das Wort »Zwangsarbeit « wird im Deutschen zur Bezeichnung von staatlichen Strafmaßnahmen erst seit dem früheren 19. Jahrhundert geläufiger: Johann C. Lede, Rußland, in: Neckar-Zeitung Nr. 275, 8. Oktober 1826, S. 1143; Jacob Grimm, Willhelm Grimm (Hrsg.), Deutsches Wörterbuch, Bd. 1, Leipzig 1854, Sp. 545 sv. »Arbeitsstrafe«; Pierer's Universallexicon, Altenburg ${ }^{4} 1857-1865$, Bd. 1, S. 700, Bd. 2, S. 314-315, Bd. 3, S. 473-474.561, Bd. 4, S. 1-12, Bd. 6, S. 571-601, Bd. 8, S. 244 , Bd. 10, S. 11.341 , Bd. 14, S. 903-928, Bd. 16, S. 171-188, Bd. 15, S. 866-875.548-571, Bd. 18, S. 18-66.524. Das deutet vermutlich nicht nur auf neue Praktiken, sondern auch auf eine andere Vorstellung von Staat und Arbeit im industriellen Zeitalter hin. Ein früherer Beleg aus dem Jahre 1760 bezieht sich auf die häusliche Kindeserziehung: Friedrich W. Birnstiel, Wochenblatt zum Besten der Kinder, Berlin 1760, S. 672. Ich danke Marco Scheider, Projekt »Deutsches Wörterbuch « der Berlin-Brandenburgischen Akademie der Wissenschaften, für seine Hinweise.

12 Rolf Gundlach, Die Zwangsumsiedlung auswärtiger Bevölkerung als Mittel ägyptischer Politik des Mittleren Reiches, Stuttgart 1994. Vorsichtiger: Bretislav Vachala, Die Kriegsgefangenen im Lichte der schriftlichen Quellen des Alten Reiches, in: Silvia Schoske (Hrsg.), Akten des Vierten Internationalen Ägyptologen Kongresses München 1985, Hamburg 1991, S. 87-93. 


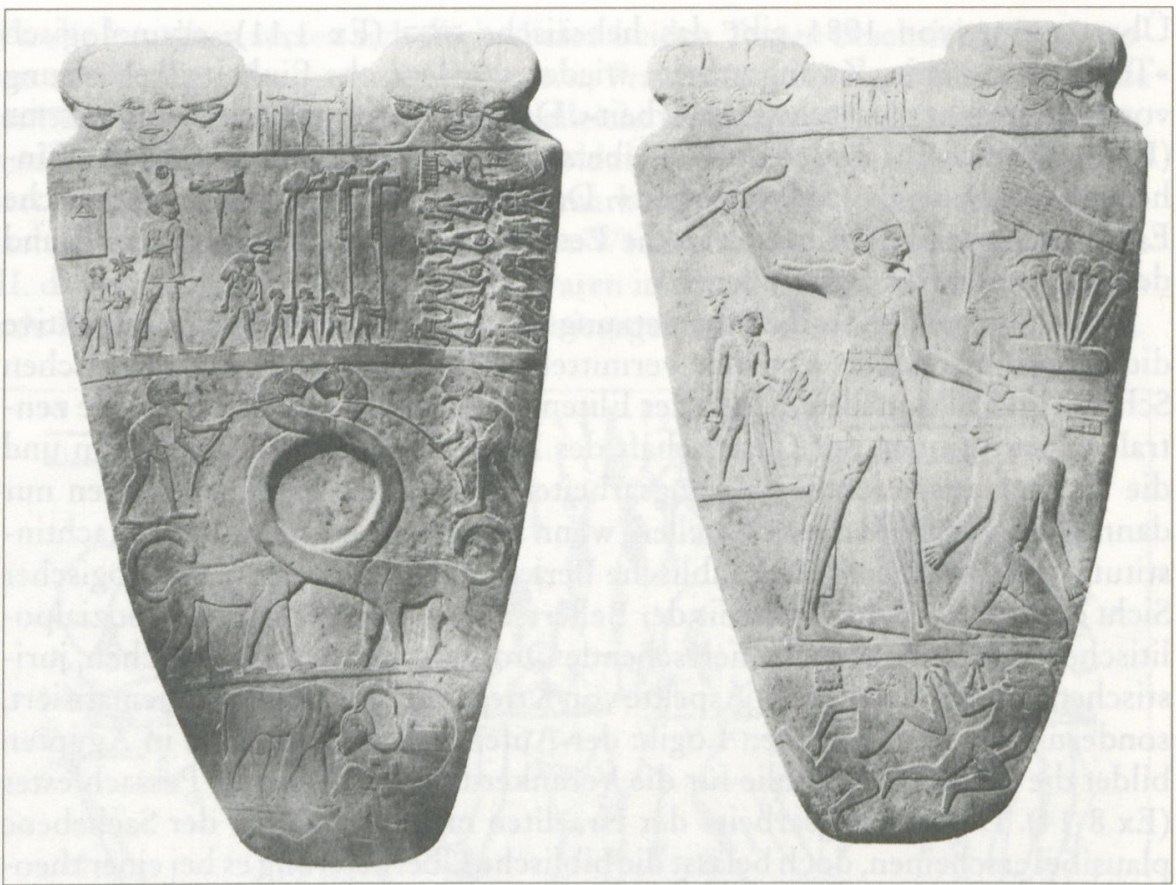

Abb. 3: Monumentale Schminkpalette des spätprädynastischen Proto-Königs Narmer, gestiftet in den Horustempel von Hierakonpolis, ca. 3300 v. Chr. Rechts: Narmer erschlägt einen Feind. Höhe: $63 \mathrm{~cm}$. Nach James Quibell, Frederik Green, Hierakonpolis I, London 1900, pl. 29.

Doch das »Niederschlagen der Feinde« mag realiter lediglich lokal begrenzte Konflikte widerspiegeln oder ist als eine ikonographische Konvention königlicher Repräsentation zu verstehen, hinter der sich durchaus friedliche Interaktionsformen mit anderen Bevölkerungsgruppen verbergen. Darüber hinaus ist die traditionelle historische Interpretation des Ikons in der Ägyptologie einer stärkeren Deutung als Medienereignis gewichen. ${ }^{13}$ Die Szene ist zwar mit anderen Darstellungen des siegreichen Königs schon in der Zeit vor dem Neuen Reich prominent auf Prestigeobjekten dargestellt worden, so in der Innendekoration der königlichen Totentempel oder in großen Felsinschriftentableaus. Im Neuen Reich aber verschob sich der Sinn des Ikons vom Kampf hin zum Krieg im größeren Maßstab. Die Szene ist jetzt monumental und weithin sichtbar an den Eingangstürmen der Tempel angebracht, und großflä-

13 Silvia Schoske, Das Erschlagen der Feinde. Ikonographie und Stilistik der Feindvernichtung im Alten Ägypten, Ann Arbor 1994; Marcus Müller, Bildliche Quellen zur Militärgeschichte, in: Gundlach/Vogel (Hrsg.), Militärgeschichte, S. 217-242; Michaela Luiselli, The Ancient Egyptian scene of $» P h a r a o h$ smiting his enemies $\alpha$. An attempt to visualize cultural memory?, in: Martin Bommas (Hrsg.), Cultural Memory and Identity in Ancient Societies, London/New York 2011, S. 10-25. 
chige Schlachtenreliefs zieren die Wände der Tempelhöfe (Abb. 4). ${ }^{14}$ Zusammen mit königlichen Kriegsberichten und archäologischen Befunden außerhalb Ägyptens zeugen sie von einer imperialistischen Hegemonialstrategie der Pharaonen. ${ }^{15}$

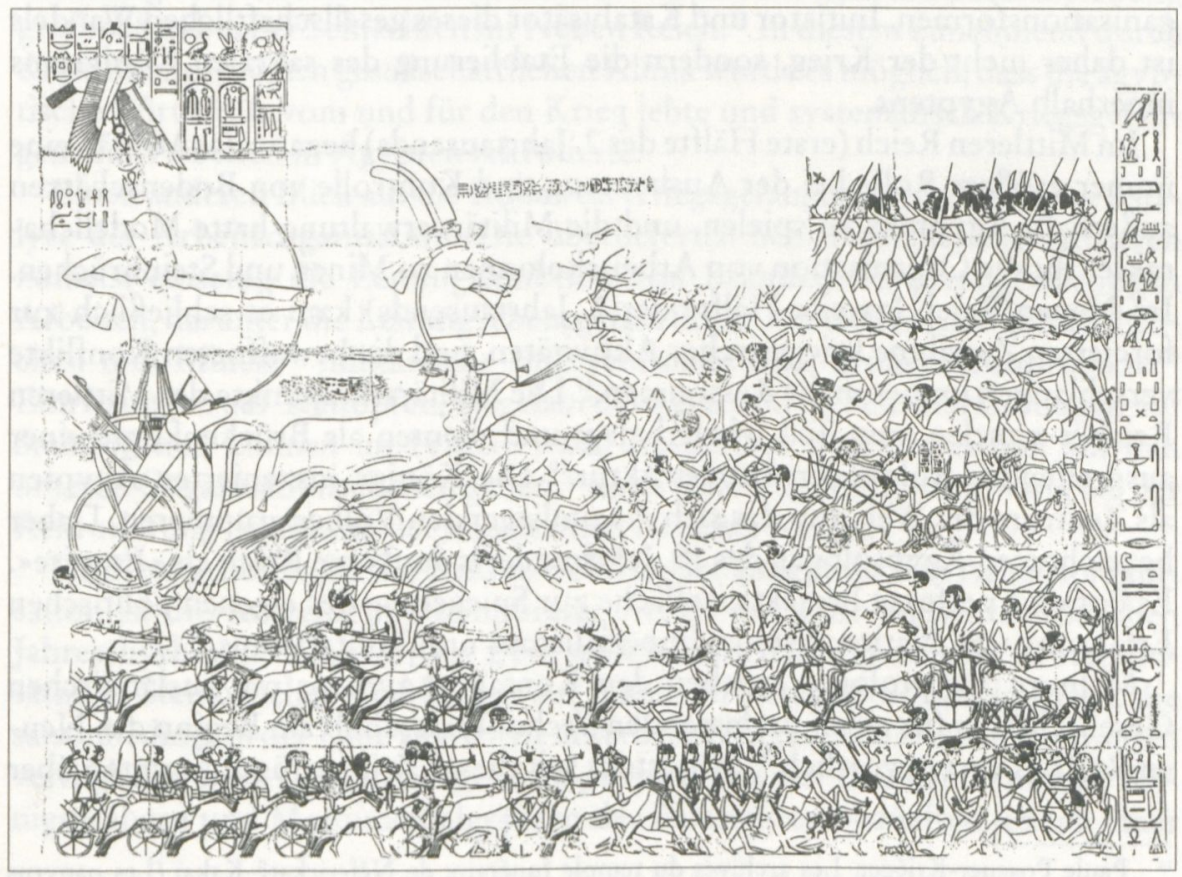

Abb. 4: Schlachtendarstellung des Totentempels Ramses' III. (1182-1151 v. Chr.) in Medinet Habu, Innenseite der Ostwand des 1. Hofes. Nach Susanne C. Heinz, Die Feldzugsdarstellungen des Neuen Reichs: Eine Bildanalyse, Wien 2001, S. 312, Katalognummer I.29.

Die Ursprünge großmaßstäblicher Arbeitsorganisation in Ägypten sind daher nicht im Militärischen zu suchen. Vielmehr ist der königliche Totenkult die treibende Kraft der sozioökonomischen Entwicklung des Alten Reiches (3. Jahrtausend). Der Bau und Betrieb der Pyramiden erforderte technologisches Know-how zur Beschaffung und Bearbeitung der nötigen Materialien sowie logistisches Wissen zur Koordination und Versorgung zahlreicher Arbeiter. Die Verwaltungsakten der königlichen Totentempel des Alten Reiches geben

\footnotetext{
Susanna C. Heinz, Die Feldzugsdarstellungen des Neuen Reichs: Eine Bildanalyse, Wien 2001.

15 Kritisch zum Imperialismusbegriff: Barry J. Kemp, Imperialism and Empire in the New Kingdom Egypt, in: P. D. A. Garnsey/C. R. Whittaker (Hgg.), Imperialism in the Ancient World, Cambridge 1978, S. 7-57; Stuart Tyson Smith, Askut in Nubia. The economics and ideology of Egyptian imperialism in the second millennium B.C., London, New York 1995, S. 1-24 und 180-188; zuletzt Ellen Fowles Morris, The architecture of imperialism. Military bases and the evolution of foreign policy in Egypt's New Kingdom, Leiden 2005, S. 1-26.
} 
Einblick in ein komplexes System von rotierenden Belegmannschaften von Priestern (»Phylen«), deren Unterhalt von landesweit eingetriebenen Abgaben bestritten wurde. ${ }^{16}$ Der königliche Totenkult und die Monumentalrepräsentation des Königtums führten zur Binnenkolonisation Ägyptens, zur verstärkten Ausbeutung von Bodenschätzen und zu komplexer werdenden sozialen Organisationsformen. Initiator und Katalysator dieses gesellschaftlichen Wandels ist daher nicht der Krieg, sondern die Etablierung des sakralen Königtums innerhalb Ägyptens.

Im Mittleren Reich (erste Hälfte des 2. Jahrtausends) begann das Militär, eine immer größere Rolle bei der Ausbeutung und Kontrolle von Bodenschätzen außerhalb Ägyptens zu spielen, und die Militärverwaltung hatte Modellcharakter für die Organisation von Arbeitskolonnen zu Minen und Steinbrüchen. Im Neuen Reich (zweite Hälfte des 2. Jahrtausends) kam es schließlich zur forcierten Zunahme kriegerischer Aktivitäten, und die bewaffneten Konflikte verschlangen immer mehr Ressourcen. ${ }^{17}$ Die Militärstützpunkte des Mittleren Reiches wurden zum Teil wiederbelegt und dienten als Brückenköpfe einer ausgreifenden kolonialen Infrastruktur. ${ }^{18}$ Die Könige inszenierten Ägypten als Zentrum der Welt, um das sich die umliegenden Völker gruppieren. Daher bezeichneten Ägyptologen des 19. Jahrhunderts das Neue Reich als »Empire«. Es bot eine perfekte Projektionsfläche zur Spiegelung der eigenen politischen Erfahrung von Nationalismus, Imperialismus und Militarismus. ${ }^{19}$

Mehrere Ägyptologen machen den Kontakt Ägyptens mit ausländischen Gesellschaften für einen umfassenden Technologieschub am Beginn des Neuen Reiches verantwortlich. ${ }^{20}$ Spätestens seit dieser Zeit verfügte Ägypten über

16 Paule Posener-Kriéger, Les archives du temple funéraire de Néferirkarê-Kakaï (Les papyrus d'Abousir), Kairo 1976; Paule Posener-Kriéger/Miroslav Verner/Hana Vymazalová, Abusir X. The Pyramid Complex of Raneferef. The Papyrus Archive, Prag 2006; Ann Macy Roth, Egyptian Phyles in the Old Kingdom. The Evolution of a System of Social Organization, Chicago 1991, S. 41-60 und 205-216.

17 Philip Gilbert, Weapons, warriors and warfare in early Egypt, Oxford 2004; Andrea M. Gnirs, Militär und Gesellschaft. Ein Beitrag zur Sozialgeschichte des Neuen Reiches. Heidelberg 1996; Anthony J. Spalinger, War in Ancient Egypt, Oxford, Malden/Carlton 2005; Rolf Gundlach/ Carola Vogel (Hrsg.), Militärgeschichte des pharaonischen Ägypten. Altägypten und seine Nachbarkulturen im Spiegel aktueller Forschungen, Paderborn 2009.

18 Stuart T. Smith, State and Empire in the Middle and New Kingdoms, in: Judith Lustig (Hrsg.), Egyptology and Anthropology. A developing dialogue, Sheffield 1997, S. 66-89, hier S. 66-68.

19 Thomas Schneider, Periodizing History: Manetho, Convention, and Beyond, in: Klaus-Peter Adam (Hrsg.), Historiographie in der Antike, Berlin/New York 2008, S. 181-195, hier S. 182. Der europäische Imperialismus spielte auch jenseits der Geschichtsschreibung eine konstitutive Rolle bei der Entstehung der »modernen Ägyptologie«. Vgl. David Jeffreys (Hrsg.), Views of Ancient Egypt since Napoleon Bonaparte. Imperialism, Colonialism and Modern Appropriations, London 2003.

20 Ian Shaw, Egyptians, Hyksos and Military Technology. Causes, Effects or Catalysts?, in: Andrew J. Shortland (Hrsg.), The Social Context of Technological Change. Egypt and the Near East, 1650-1550 BC, Oxford 2001, S. 59-72; P. R. S. Moorey, The Mobility of Artisans and Opportunities for Technology Transfer Between Western Asia and Egypt in the Late Bronze Age, in: ebd., S, 15-38, hier S. 12. Vgl. dagegen auch Heike Wilde, Innovation und Tradition. Zur Herstellung und Verwendung von Prestigegütern im pharaonischen Ägypten, Wiesbaden 2011, S. 243. 
ein stehendes Heer. Hier boten sich neue Möglichkeiten des sozialen Aufstiegs. ${ }^{21}$ Hohe Militärs übernahmen zunehmend gesellschaftliche Führungspositionen, und die Militärverwaltung wurde zum Vorbild für die Strukturierung ziviler Verwaltungszweige. ${ }^{22}$ Auch in der Literatur Ägyptens zeigt sich eine Wendung vom heroischen Zweikampf im Mittleren Reich hin zur Darstellung großmaßstäblicher Schlachten im Neuen Reich. ${ }^{23}$ In diesem zunehmend durch das Militär geprägten gesellschaftlichen Klima wurde es möglich, dass die ägyptische Wirtschaft vom und für den Krieg lebte und systematisch Kriegsgefangene für Arbeiten in Ägypten rekrutierte.

Einen anderen Blick auf die Rolle von Kriegsgefangenen erschließt die Analyse der Arbeitsorganisation. Die überlieferten Schriftquellen thematisieren zumeist einseitig die Leitungsfunktion von Beamten bei prestigeträchtigen Arbeiten, darunter die Leitung lebensgefährlicher Arbeitseinsätze in Steinbrüchen und Minen, ${ }^{24}$ militärische Interventionen oder größere Bauprojekte. ${ }^{25}$ Einritzungen auf Tontöpfen, Siegelabrollungen und Arbeitsmarkierungen auf Steinblöcken können im Prinzip zwar Aufschluss über die Arbeit auf den unteren Organisationsebenen geben ${ }^{26}$ doch bleibt die Rolle von $Z$ wang bei der Rekrutierung der Masse der ungelernten Arbeiter unklar. ${ }^{27}$

Ein Kernproblem für die Rekonstruktion der altägyptischen Arbeitsorganisation ist die Korrelierung temporärer Arbeit mit dem landwirtschaftlichen Jahresrhythmus. ${ }^{28}$ Christopher Eyre stellt heraus, dass die meisten Arbeitseinsätze in Steinbrüchen und Minen auf lokale Arbeitskräfte zurückgriffen, die saisonbedingt in der Landwirtschaft unterbeschäftigt und als Arbeitsmigranten verfügbar waren. Längerfristige Großprojekte wie der Bau von Tempeln, Königsgräbern und Monumentalgräbern der Palasteliten bedürften jedoch einer

21 Spalinger, War, S. 25-26 und 27, Anm. 4.

22 Gnirs, Krieg und Gesellschaft, S. 41-191; Spalinger, War, S. 264-277.

23 Andrea M. Gnirs/Antonio Loprieno, Krieg und Literatur, in: Gundlach/Vogel (Hrsg.), Militärgeschichte, S. 243-308.

24 Elizabeth Bloxam, Quarrying and Mining (Stone), in: Willeke Wendrich (Hrsg.), UCLA Encyclopedia of Egyptology, Los Angeles 2010. Eine Stele Ramses' IV. listet Todesfälle unter den Arbeitern auf: Vgl. Louis Christophe, La stèle de l'an III de Ramsès IV au Ouâdi Hammâmât no. 12, in: Bulletin de l'Institut Français d'Archéologie Orientale 48 (1949), S. 1-38.

25 Der Abschnitt stützt sich im Wesentlichen auf die Arbeiten von Christopher Eyre: Work and the organisation of work in the Old Kingdom, in: Marvin A. Powell (Hrsg.), Labor in the Ancient Near East, New Haven 1987, S. 5-47; ders., Work and the organisation of work in the New Kingdom, in: ebd., S. 167-221. Außerdem: Bernadette Menu (Hrsg.), L'organisation du travail en Égypte et en Mésopotamie, Kairo 2010.

26 Zum Beispiel Petra Andrássy, Builders' graffiti and administrative aspects of pyramid and temple building in Ancient Egypt, in: René Preys (Hrsg.), 7. Ägyptologische Tempeltagung. Structuring religion, Leuven 28. September-1. Oktober 2005, Wiesbaden 2009, S. 1-16. Bernadette Menu, Quelques aspects du recrutement des travailleurs dans l'Égypte du deuxième millénaire av. J.-C., in: Menu (Hrsg.), L'organisation du travail, S. 171-183.

28 Christopher Eyre, Village Economy in Pharaonic Egypt, in: Alan K. Bowman/Eugene Rogan (Hgg.), Agriculture in Egypt. From Pharaonic to Modern Times, Oxford 1999, S. 33-60; Juan Carlos Moreno-Garcia, Hwt et le milieu rural égyptien du IIIe millénaire. Économie, administration et organisation territoriale, Paris 1999; Bernadette Menu (Hrsg.), La dépendance rurale dans l'Antiquité égyptienne et proche-orient, Kairo 2001. Micòl di Teodoro (London) untersucht diesen Zusammenhang derzeit in einer Dissertation. 
größeren Zahl von Arbeitskräften, die - zumindest im Neuen Reich - nur durch Kriegsgefangene hätte abgedeckt werden können. ${ }^{29}$ Diese Entwicklung hin zu neuen Formen der Arbeitsrekrutierung war in Ägypten damit das Ergebnis dreier gesellschaftlicher Basisprozesse: (1) der Etablierung der sesshaften Landwirtschaft im Laufe des Neolithikums (ca. 8000-4000 v. Chr.), ${ }^{30}$ (2) des Aufstiegs des sakralen Königtums und dessen Monumentalrepräsentation in einer komplexer werdenden Gesellschaft, und (3) der zunehmenden Militarisierung der Gesellschaft.

Als besonders problematisch bei der Definition und Untersuchung von Zwangsarbeit in Ägypten erweist sich die Bestimmung des Aspekts des "Zwangs«. Zwang kann von der gezwungenen Person als Form der Gewalt empfunden, von der erzwingenden aber als legitim betrachtet und von Außenstehenden völlig übersehen werden. Hinzu kommt, dass der Konsens über das Zulässige in einer Gesellschaft in der Regel umstritten ist. Historiker können über das verfügbare Quellenmaterial oftmals jedoch nur einseitig die Sichtweise der Herrschenden fassen. Die rechtliche Abgrenzung von Zwangsarbeit gegenüber Sklaverei oder Erwerbsarbeit ist im pharaonischen Ägypten wegen der schmalen Quellenbasis lediglich annäherungsweise möglich. ${ }^{31}$ Zwar gibt es einen Diskurs über Gerechtigkeit in Ägypten, doch nicht über Arbeitsrecht, Bürgerrechte oder Disziplinarmaßnahmen. ${ }^{32}$ Unklar ist zudem, welche soziokulturelle Bedeutung die Arbeit im alten Ägypten hatte und ob sie ähnlich wie heute als vermittelnde Größe zwischen Individuum und Gesellschaft thematisiert wurde. ${ }^{33}$

Ähnlich problematisch ist eine kulturspezifische Definition von Gewalt. ${ }^{34}$ Gewalt, Zwang und Konflikt werden in Ägypten außerhalb der königlichen

29 Eyre, Work in the Old Kingdom, in: Powell (Hrsg.), Labor, hier S. 15-20; ders., Work in the New Kingdom, in: Powell (Hrsg.), Labor, hier S. 180-183 und 188-190; ders., Who built the great temples of Egypt?, in: Menu (Hrsg.), L'organisation du travail, S. 117-138, hier S. 132138; Barry J. Kemp, Ancient Egypt. Anatomy of a civilization, London/New York ${ }^{2} 2006$, S. 26-33.

30 Noriyuki Shirai, The archaeology of the first farmer-herders in Egypt. New insights into the Fayum Epipalaeolithic and Neolithic, Leiden 2010, hier S. 22-24; David Wengrow, The Archaeology of Early Egypt. Social Transformations in North-East Africa 10,000 to 2650 BC, Cambridge 2006, S. 41-71.

31 Sandra Lippert, Einführung in die altägyptische Rechtsgeschichte, Münster 2008, zu »Sklaven * und "Arbeitsverpflichtungen « S. 12-14, 34-35, 55-57 und 112-115.

32 Jan Assmann, Ma'at. Gerechtigkeit und Unsterblichkeit im Alten Ägypten, München 1990; Andrea M. Gnirs (Hrsg.), Reading the Eloquent Peasant, Göttingen 2001.

33 Helmuth Schneider weist in der Einleitung zu Arne Eggebrecht u.a., Geschichte der Arbeit. Vom Alten Ägypten bis zur Gegenwart, Köln 1980, hier S. 12 auf den Zusammenhang von aufkommendem Frühkapitalimus und der theoretischen Objektivierung von »Arbeit« hin. Er schlägt aber wohl zu Unrecht eine seit der Antike ungebrochene Diskursgeschichte des Begriffes vor. Für eine philologische Annäherung an Bedeutungsdimensionen von »Arbeit « in Ägypten vgl. Katalin A. Kóthay, La notion de travail au Moyen Empire. Implications sociales, in: Menu (Hrsg.), Organisation de travail, S. 155-170.

${ }^{34}$ Vgl. die Diskussion bei Hubert Röder, Der Nedjti zwischen Kriegszug und Vernichtungsopfer. Potenzielle Räume institutionalisierter Grausamkeit im Alten Ägypten, in: Trutz von Trotha/ Jakob Rösel (Hrsg.), On Cruelty - Sur la cruauté - Über Grausamkeit, Siegener Beiträge zur Soziologie 11, Köln 2011, S. 285-353, hier S. 285-301. 
Kriegsszenen nur schwach thematisiert. ${ }^{35}$ Auf den ansonsten reich bebilderten Grabwänden der Eliten wird dieser Themenkomplex fast vollständig ausgeblendet und gemieden. ${ }^{36}$ Archäologisch weist der Knochenbefund von Bestattungen, insbesondere der sozial Benachteiligten, etwa von Frauen, aber durchaus auf häusliche Gewalt und auf die Verrichtung schwerer körperlicher Arbeit hin. ${ }^{37}$

Methodisch ist die Archäologie insgesamt ein wichtiger Schlüssel, um die Konstruktion des Elitendiskurses zu verstehen, da sie stärker als Schrift- und Bildquellen auch Bevölkerungsgruppen jenseits der Verwaltungseliten abbildet. Eine wichtige Rolle bei der archäologischen Erforschung von Zwangsarbeit und der damit verbundenen Aspekte von Überwachung und Normierung spielen in der Ägyptologie Plansiedlungen. Sie entstanden im Zusammenhang mit königlichen Großprojekten, so beim Bau der Pyramiden, im Tal der Könige und in der neu geplanten Hauptstadt Echnatons "Akhetaton «, sowie in Expeditionsgebieten der Ostwüste und im nubischen Grenzgebiet. ${ }^{38}$

Eine der bekanntesten Plansiedlungen ist die Pyramidenstadt Sesostris' II. aus dem Mittleren Reich beim heutigen Kahun/el-Lahun (Abb. 5). ${ }^{39}$ Sie beherbergte zahlreiche Priester, Funktionäre und Arbeiter, darunter Ägypter und "(Vorder)-Asiaten ${ }^{40}{ }^{40}$ die entweder als Kriegsgefangene oder als Nachkommen ehemaliger Einwanderer beim Bau der Pyramide und der Höflingsgräber zum Einsatz kamen. Das Gros der in Kahun lebenden ungelernten Arbeiter dürfte zum Steine schleppen und in der Landwirtschaft eingesetzt gewesen sein. In den erhaltenen Papyrusdokumenten von Kahun tauchen sie jedoch nur als Verwaltungsmasse auf, weil die Akten vor allem auf Personen mit Spezialberufen fokussieren.

35 Vgl. jedoch Renate Müller-Wollermann, Vergehen und Strafen. Zur Sanktionierung abweichenden Verhaltens im Alten Ägypten, Leiden/Boston 2004.

36 John Baines, Society, Morality, and Religious Practice, in: Byron Shafer (Hrsg.), Religion in Ancient Egypt. Gods, Myths, and Personal Practice, London 1991, S. 123-200, hier S. 138-139 mit Anmerkungen auf S. 44 und 45.

37 Stephan J. Seidlmayer, Archäologische Befunde militärgeschichtlicher Aussagekraft, in: Gundlach/Vogel (Hrsg.), Militärgeschichte, S. 147-164, hier S. 152.

38 Richard Bußmann, Siedlungen im Kontext der Pyramiden des Alten Reiches, in: Mitteilungen des Deutschen Archäologischen Instituts, Abteilung Kairo 60 (2004), S. 19-37; Carola Vogel, Ägyptische Festungen und Garnisonen bis zum Ende des Mittleren Reiches, Hildesheim 2004; Barry J. Kemp, The Amarna workmen's village in retrospect, in: Journal of Egyptian Archaeology 73 (1987), S. 21-50; Kemp, Ancient Egypt, S. 211-244; Mark Lehner, Villages and the Old Kingdom, in: Wendrich (Hg.), Egyptian Archaeology, Malden/Oxford/Chichester 2010, S. 85101; Nadine Moeller, The influence of royal power on Ancient Egyptian settlements, in: Cahiers de Recherches de l'Institut de Papyrologie et d'Égyptologie de Lille 28 (2009-2010), 193-210, hier S. 202-207.

39 Zusammenfassend Stephen Quirke, Lahun. A town in Egypt $1800 \mathrm{BC}$ and the history of its landscape, London 2005. Zuletzt Florence Doyen, La résidence d'élite. Un Type de structure dans l'organisation spatiale urbaine du Moyen Empire, in: Mafred Bietak/Ernst Czerny/Irene Forstner-Müller (Hrsg.), Cities and Urbanism in Ancient Egypt, Wien 2010, S. 81-101.

40 Ulrich Luft, Asiatics in Illahun. A preliminary report, in: International Association of Egyptologists (Hrsg.), Atti. Sesto Congresso Internazionale di Egittologia 2, Turin 1993, S. 291-297. Die konventionelle ägyptologische Übersetzung des ägyptischen Ethnonyms aamu als »Asiat « stützt sich auf die semitischen Namen von Personen, die dieser Bevölkerungsgruppe angehören. Vermutlich handelt es sich um Gruppen, die im heutigen Palästina und der Levante siedelten. 


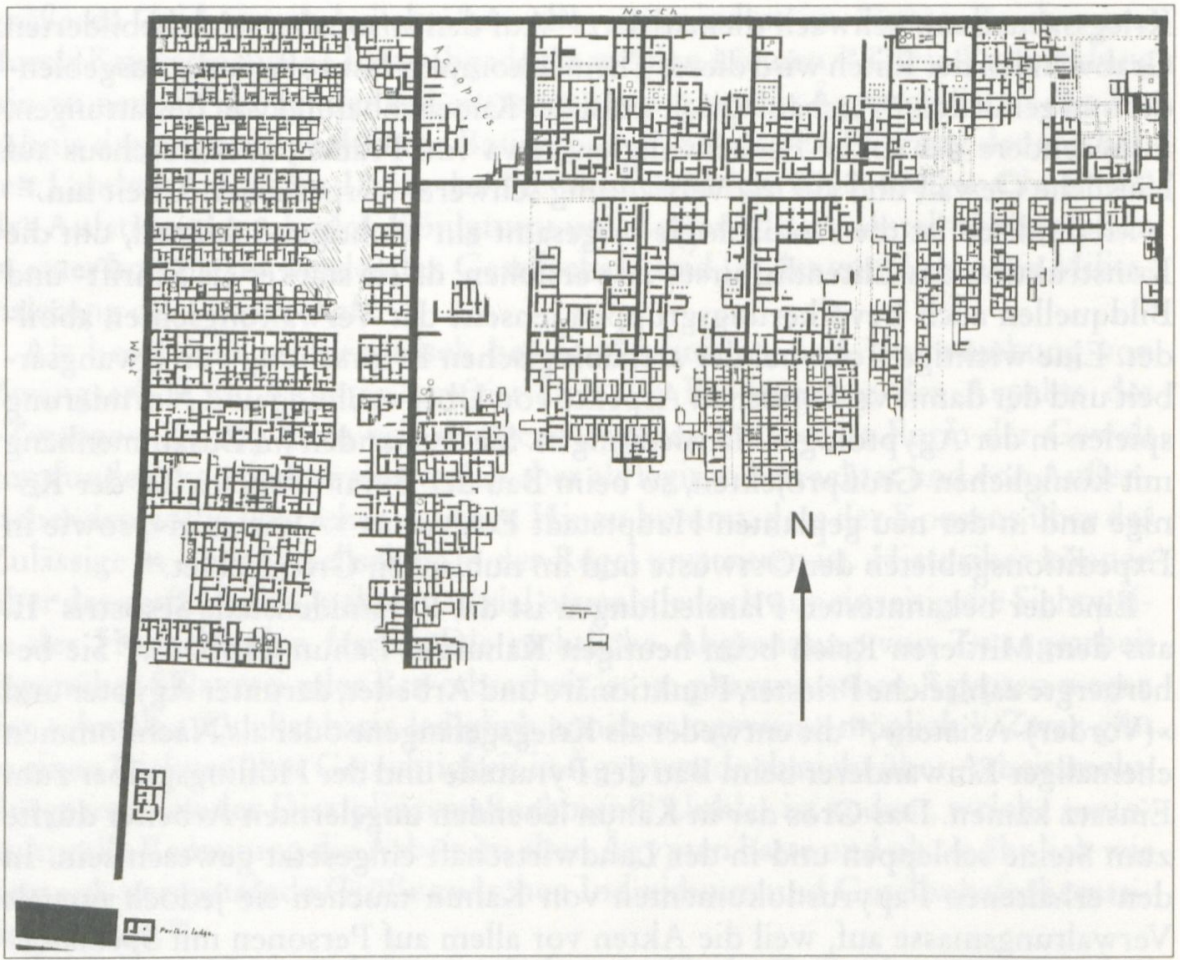

Abb. 5: Geplante Pyramidenstadt Sesostris' II. bei Kahun, ca. 1800 v. Chr., ca. 400 m x 400 m. Nach W. M. Flinders Petrie, Illahun, Kahun, and Gurob, London 1891, pl. 14 .

Jüngere Ausgrabungen geben eine Vorstellung davon, inwieweit Plansiedlungen wie Kahun von »normalen « Siedlungen ihrer Zeit abwichen. Im Unterschied zur gewachsenen Provinzstadt des Mittleren Reiches auf der Insel Elephantine (Abb. 6) ${ }^{41}$ hatte Kahun eine orthogonale Struktur mit normierten Hausgrundrissen, die sich nicht an der zeitgenössisch üblichen Gestaltung von Privatsphäre und Öffentlichkeit orientierten. Die Siedlung wurde am Reißbrett entworfen und drängte den Bewohnern eine vorgegebene Gemeinschaftsstruktur auf. Formen des Zwangs und der Kontrolle scheinen sich so in die Siedlungsstruktur einzuschreiben. Anders als in den nubischen Militärkasernen konnten die Bewohner Kahuns aber die Häuser mit Umbauten ihren Wohnbedürfnissen anpassen. Sie veränderten die von den Verwaltungseliten entworfene Visionen der Raumgestaltung und nahmen Einfluss auf die soziale Organisation von Alltag und Arbeit. Das deutet darauf hin, dass Kahun zwar eine Funktionssiedlung, aber kein Arbeits-, Bestrafungs- oder Erziehungslager für Zwangsarbeiter war, das einer rigiden Ordnung unterworfen war.

${ }^{41}$ Cornelius von Pilgrim, Elephantine 18. Untersuchungen in der Stadt des Mittleren Reiches und der Zweiten Zwischenzeit, Mainz 1996. 


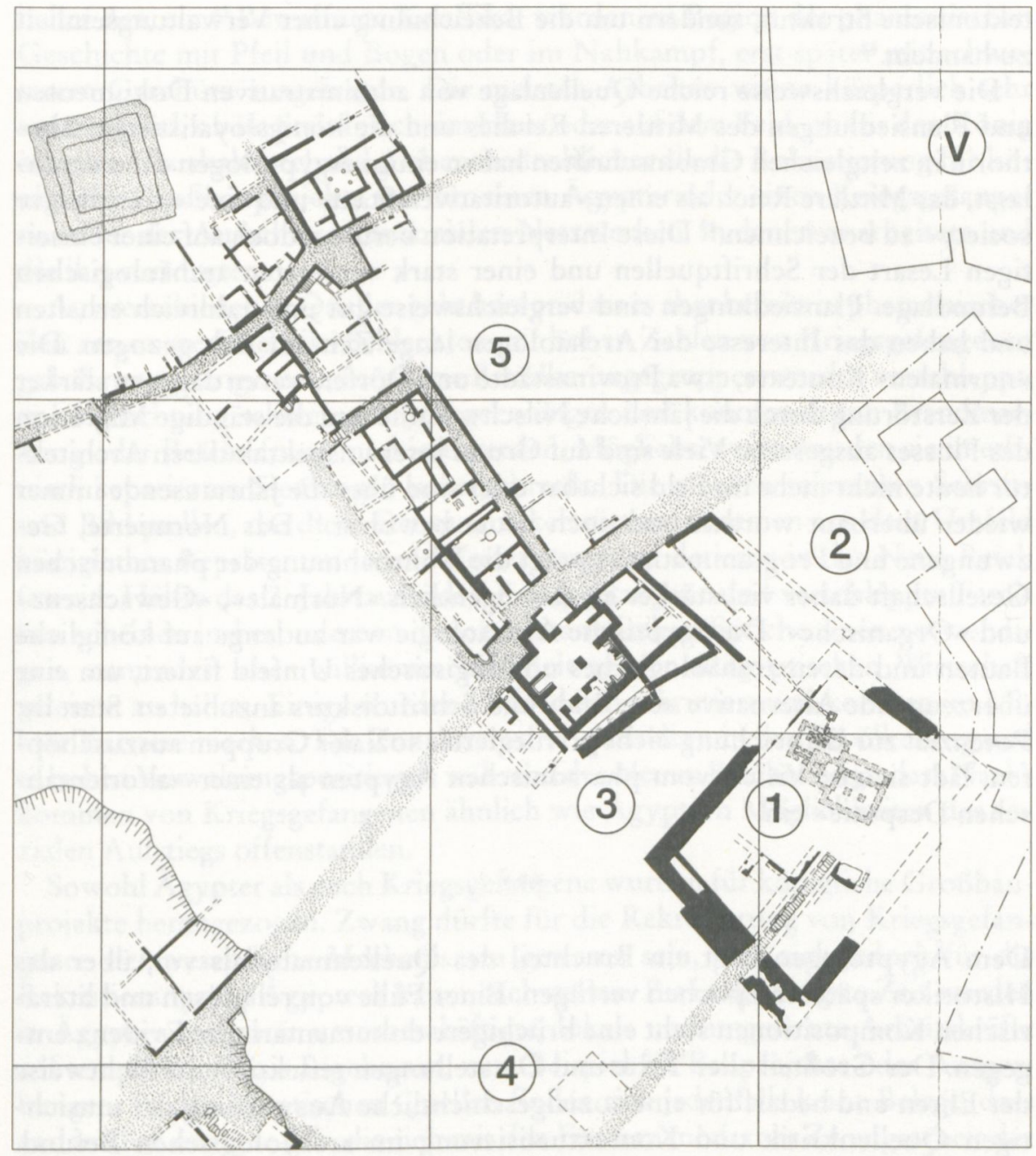

Abb. 6: Ausgegrabener Streifen der Siedlung des späten Mittleren Reiches von Elephantine bei (5), ca. 1700 v. Chr. Quadranten $10 \mathrm{~m}$ x 10m. Nach Werner Kaiser et al., Stadt und Tempel von Elephantine. 25./26./27. Grabungsbericht, in: Mitteilungen des Deutschen Archäologischen Instituts, Abteilung Kairo 55 (1999), Abb. 56

Gleichwohl kann nicht ausgeschlossen werden, dass die Arbeit in Kahun (auch) unter Zwang verrichtet werden musste. Ein zeitgleicher, vermutlich aus Oberägypten stammender Papyrus wird als Liste von Arbeitsdeserteuren gedeutet, die dem »chenret wer « entflohen waren. Der »chenret wer « wird auch in den Verwaltungsakten von Kahun erwähnt. Ursprünglich annährungsweise als »Gefängnis« oder »Lager " gedeutet und mit Kahun assoziiert, scheint es sich bei diesem Begriff neueren Forschungen nach aber nicht um eine archi- 
tektonische Struktur, sondern um die Bezeichnung einer Verwaltungseinheit zu handeln. ${ }^{42}$

Die vergleichsweise reiche Quellenlage von administrativen Dokumenten und Plansiedlungen des Mittleren Reiches und die königsloyalistische Rhethorik in zeitgleichen Grabinschriften haben einige Ägyptologen dazu veranlasst, das Mittlere Reich als einen »autoritativen Staat« und eine »prescriptive society « zu bezeichnen. ${ }^{43}$ Diese Interpretation beruht jedoch auf einer einseitigen Lesart der Schriftquellen und einer stark verzerrten archäologischen Befundlage. Plansiedlungen sind vergleichsweise gut und zahlreich erhalten und haben das Interesse der Archäologen lange Zeit auf sich gezogen. Die »normalen « Kontexte, etwa Provinzstädte und Dörfer, waren dagegen stärker der Zerstörung durch die jährliche Nilschwemme und die ständige Migration des Flusses ausgesetzt. Viele sind auf Grund ihrer unspektakulären Architektur heute nicht mehr im Feld sichtbar oder sind über die Jahrtausende immer wieder überbaut worden und noch heute bewohnt. ${ }^{44}$ Das Normierte, Gezwungene und Programmatische prägt die Wahrnehmung der pharaonischen Gesellschaft daher viel stärker als das eigentlich "Normale", "Gewachsene" und »Organische«. Die ägyptische Archäologie war zu lange auf königliche Bauten und deren archäologisches und logistisches Umfeld fixiert, um eine überzeugende Alternative zum Bild- und Schriftdiskurs anzubieten. Statt ihr Potenzial zur Erforschung nicht privilegierter sozialer Gruppen auszuschöpfen, lädt sie zur Vision vom pharaonischen Ägypten als einer »altorientalischen Despotie« ein.

$* * *$

Dem Ägyptologen liegt ein Bruchteil des Quellenmaterials vor, über das Historiker späterer Epochen verfügen. Einer Fülle von religiösen und literarischen Kompositionen steht eine brüchigere dokumentarische Evidenz entgegen. Der Großteil aller Texte und Darstellungen reflektiert die Sichtweise der Eliten und bedarf für eine sozialgeschichtliche Auswertung der umsichtigen Quellenkritik und Kontextualisierung im archäologischen Befund. Neuzeitliche Begriffe wie Staat, Krieg und Zwangsarbeit dürfen nicht darüber hinwegtäuschen, dass das alte Ägypten im Kern eine face-to-face Gesellschaft mit einer dünnen Schriftbürokratie war. Letztere dominiert zwar im Quellenmaterial, drang aber de facto nur schwach in verbindlichere Formen von Loyalität und sozialer Abhängigkeit, insbesondere verwandtschaft-

42 William C. Hayes, A Papyrus of The Late Middle Kingdom in the Brooklyn Museum. Papyrus Brooklyn 35.1446, New York 1955, hier S. 34-58; S. Quirke, State and Labour in the Middle Kingdom: A Reconsideration of the term xnrt, in: Revue d'Égyptologie 39 (1988), S. 83-106; Joachim Sliwa, Der bnrt von Qasr el-Sagha, in: Peter Jánosi (Hrsg.), Structure and Significance. Thoughts on Ancient Egyptian Architecture, Wien 2005, S. 477-483.

43 Kemp, Ancient Egypt, S. 241-244; Jan Assmann, Ägypten. Eine Sinngeschichte, München/Wien 1996, S. 135-222.

44 Manfred Bietak, Urban Archaeology and the »Town Problem « in Ancient Egypt, in: Kent Weeks (Hrsg.), Egyptology and the social sciences. Five studies, Kairo 1979, S. 97-144. 
licher Art, ein. ${ }^{45}$ Bewaffnete Konflikte wurden zu Beginn der pharaonischen Geschichte mit Pfeil und Bogen oder im Nahkampf, erst später mit schwererem Geschütz ausgetragen. Die meisten Arbeiten waren körperlich sehr anstrengend, ob im Steinbruch, im Haus oder auf dem Feld, ob für den König oder im Haushaltsverband. Lebensbedrohlicher als die Rekrutierung für königliche Großbauprojekte war für einen Ägypter oder einen Kriegsgefangenen aber der Ausschluss aus sozialen Netzwerken, in denen er arbeitete und die ihn versorgten.

Aus verschiedenen Quellen geht hervor, dass es aber bereits in pharaonischer Zeit ressourcenintensive Schlachten mit hohen Zahlen von Kriegsgefangenen gab. Kriegsgefangene und eroberte Bevölkerungsgruppen wurden verschleppt, zur Verrichtung schwerer Arbeit gezwungen und konnten durch Brandmarkung oder Beschriftung markiert werden. Möglicherweise wurden sie in ethnisch separierten Siedlungen untergebracht. Die meisten relevanten Schriftund Bildquellen, die diese Deutung unterstützen, stammen aus dem Umfeld königlicher Repräsentation und Verwaltung und datieren in das Neue Reich (zweite Hälfte des 2. Jahrtausends). Kriegsgefangene und verschleppte Familien scheinen insbesondere in dieser militaristischen Epoche einen notwendigen, zumindest aber willkommenen Beitrag zur altägyptischen Wirtschaft geleistet zu haben. Einige wurden als Soldaten rekrutiert und konnten im Militär Karriere machen. Die Zunahme von Ausländern in der Bevölkerung und in hohen Verwaltungspositionen während des Neuen Reiches zeigt, dass Nachkommen von Kriegsgefangenen ähnlich wie Ägyptern Möglichkeiten des sozialen Aufstiegs offenstanden.

Sowohl Ägypter als auch Kriegsgefangene wurden für königliche Großbauprojekte herangezogen. Zwang dürfte für die Rekrutierung von Kriegsgefangenen der wesentliche Mechanismus gewesen sein, lässt sich jedoch für die Rekrutierung von Ägyptern kaum nachweisen. Bis in die jüngste Zeit werden in Ägypten temporär unterbeschäftigte, lokale und ungelernte Arbeitskräfte auf temporärer Basis angeheuert, zum Beispiel für Bauprojekte oder Ausgrabungen. In den ägyptischen Quellen finden sich jedenfalls keine Belege, dass es damals Konzepte gab, die sich mit den Formen moderner Zwangsarbeit des 20. Jahrhunderts wie die "Vernichtung durch Arbeit « oder die »Erziehung durch Arbeit « vergleichen lassen. Umbauten in Plansiedlungen wie der Pyramidenstadt Kahun, die auf den ersten Blick wie ein Zwangslager von Kriegsgefangenen für den Pyramidenbau aussieht, zeugen davon, dass die Bewohner die normierten Hausgrundrisse im Laufe der Zeit ihren Wohnbedürfnissen angepasst haben. Die Verwaltungsakten der Stadt geben zwar Aufschluss über ökonomische Abhängigkeiten ägyptischer und nicht-ägyptischer Personen von übergeordneten Verwaltungsinstanzen, doch weder der Stadtgrundriss noch die Akten weisen Anzeichen einer totalen Überwachung der hier lebenden Familienhaushalte auf.

45 Christopher Eyre, On the Inefficiency of Bureaucracy, in: Patrizia Piacentini/Christian Orsenigo (Hrsg.), Egyptian Archives. Proceedings of the First Session of the International Congress Egyptian Archives/Egyptological Archives, Milano, September 9-10, Mailand 2009, S. 15-30. 
Besonders vielversprechend für weiterführende Überlegungen ist eine Perspektive auf Krieg und "Zwangsarbeit « als Indikatoren und Katalysatoren eines umfassenden sozialen und politischen Wandels. In der Anthropologie etwa wird Krieg in vorstaatlichen Gesellschaften als eine Triebkraft für die Entwicklung größerer sozialer Komplexität verstanden. ${ }^{46}$ Diesem Ansatz folgen zwar einige Beiträge zur Staatsentstehung in Ägypten, die meisten sehen Krieg jedoch nur als ein Werkzeug der territorialen Expansion und Systemstabilisierung nach innen. ${ }^{47}$ Tatsächlich ist im Alten Reich (3. Jahrtausend) nicht der Krieg, sondern der königliche Totenkult die treibende Kraft für die Ausbildung neuer administrativer Netzwerke. Die Herrschaftselite konzentriert sich auf die Etablierung des Königtums innerhalb Ägyptens und greift auf neue Formen komplexer Arbeitsorganisation zur Repräsentation des sakralen Königtums zurück. Die Rolle von Zwang bei der Rekrutierung muss jedoch mangels eindeutiger Quellenlage offen bleiben. Erst im Laufe des Mittleren und Neuen Reiches (2. Jahrtausend) avanciert das Militär zu einem zentralen Wirtschaftssektor und sozialen Referenzrahmen für die Selbstdarstellung der Eliten. Im Neuen Reich rückt die Positionierung Ägyptens als das Zentrum einer Welt, die zunehmend von militärisch agierenden Großreichen geprägt war, in den Vordergrund königlichen Interesses. Entsprechend fallen die meisten Hinweise auf Zwangsumsiedlung und Arbeitsrekrutierung von Kriegsgefangenen in diese Epoche. Motor dieser Entwicklungen ist der Aufstieg und die Transformation des sakralen Königtums. "Zwangsarbeit « ist daher nicht notwendigerweise an den Krieg gekoppelt, sondern findet epochenspezifisch in den innovativen Kontexten der königlichen Inszenierung statt.

Mit dieser Perspektive kann sich die Ägyptologie trotz empirischer und theoretischer Schwierigkeiten in Studien einbringen, denen es mit diachronen und synchronen Vergleichen um ein besseres Verständnis von Krieg und Zwangsarbeit geht. Die Kontextualisierung relevanter Befunde in übergeordneten kulturellen und gesellschaftlichen Entwicklungen stellt ein wichtiges Korrektiv gegen vorschnelle Generalisierungen wie Wittfogel's Oriental Despotism dar.

46 Vgl. Henri J. M. Claessen, War and State Formation. What is the Connection?, in: Ton Otto/ Henrik Thrane/Helle Vandkilde (Hrsg.), Warfare and Society. Archaeological and Social Anthropological Perspectives, Aarhus 2006, S. 217-226; Claus Bossen, Chiefs Made War and War Made States? War and Early State Formation in Ancient Fiji and Hawai, in: ebd., S. 237-260.

47 Marcello Campagno, In the Beginning was the War. Conflict and the Emergence of the Egyptian State, in: Stan Hendrickx/Renée F. Friedman/Krzysztof M. Ciałowicz/Marek Chłodnicki (Hrsg.), Egypt at Its Origins. Studies in Memory of Barbara Adams, Löwen/Paris/Dudley MA 2004, S. 689-703, hier S. 690 mit Anm. 2. Phillip G. Gilbert diskutiert die Koevolution sozialer Komplexität und Krieg im prädynastischen Ägypten, zeichnet das Militärische aber nicht verantwortlich für die Ausbildung organisatorischer Komplexität. Vgl. Gilbert, Weapons, warriors and warfare in early Egypt. 